\title{
Modelos de análisis y prevención de deserción estudiantil universitaria dirigidos al contexto panameño
}

\section{Models of analysis and prevention of university student dropout aimed at the Panamanian context.}

\author{
DOI: $10.46932 / \mathrm{sfjdv2n2-018}$
}

Received in: january 1st, 2020

Accepted in: March 30th, 2020

\begin{abstract}
Pablo Díaz
Universidad de Panamá, Extensión Universitaria de Soná, Facultad de Ciencias de la Educación

E-mail: pablodiazstgo@gmail.com
\end{abstract}

\author{
Alexis Tejedor De León \\ Universidad Tecnológica de Panamá, Facultad de Ingeniería Mecánica y Metalurgia \\ E-mail: alexis.tejedor@up.ac.pa \\ Luz Mariela Saavedra \\ ISAE Universidad, Veraguas, Panamá, Facultad de Administración de Empresas \\ E-mail: lmariel3179@gmail.com
}

\begin{abstract}
RESUMEN
La presente investigación consta de dos momentos; el primero, hace un análisis de la deserción estudiantil universitaria mediante un instrumento denominado CADESUN que permite fijar las causas que conducen a estudiantes a abandonar sus estudios. El diseño del cuestionario ad hoc consideró los planteamientos de diversas investigaciones para ser aplicado a una muestra estudiantil desertora (2014-2015) de una IES pública del país. El cuestionario de 40 aseveraciones fue diseñado para identificar, entre la población estudiantil, cinco categorías o factores que potencialmente pueden llevarlo a abandonar sus estudios: psicológico, sociológico, económico, organizacional y de integración-adaptación, con sus respectivas subcategorías. Analizado los resultados mediante el cuestionario autoinstructivo se pudo constatar que los factores que más inciden en el abandono estudiantil, es el Modelo Económico seguido del Organizacional. Después de conocer las causales por las cuales los estudiantes abandonan sus estudios universitarios; en el segundo momento se pretende diseñar un programa de prevención de deserción basado en Modelos Latinoamericanos que permitan a los gestores universitarios analizar los requerimientos administrativos y/o académicos y tomar las medidas de mitigación correspondientes. La identificación y análisis de diversos sistemas, modelos o programas de prevención de deserción procura facilitar la creación de un Modelo propio, por ejemplo: de monitoreo, seguimiento y orientación psicopedagógica a los estudiantes desde sus inicios en la institución hasta que culmine su carrera.
\end{abstract}

Descriptores o Palabras Clave: Educación superior, Abandono, Modelos de deserción, Prevención.

\begin{abstract}
The present research consists of two moments; the first one, makes an analysis of university student desertion by means of an instrument called CADESUN that allows to fix the causes that lead students to abandon their studies. The design of the ad hoc questionnaire considered the approaches of various researches to be applied to a sample of dropout students (2014-2015) from a public IES of the country. The 40-item questionnaire was designed to identify, among the student population, five categories or factors that could potentially lead them to abandon their studies: psychological, sociological, economic,
\end{abstract}


organizational and integration-adaptation, with their respective subcategories. After analyzing the results by means of the self-instructive questionnaire, it was found that the factors that most influence student dropout are the Economic Model followed by the Organizational Model.

After knowing the causes for which students abandon their university studies, the second step is to design a dropout prevention program based on Latin American Models that will allow university managers to analyze the administrative and/or academic requirements and take the corresponding mitigation measures. The identification and analysis of various dropout prevention systems, models or programs seeks to facilitate the creation of a model of its own, for example: monitoring, follow-up and psycho-pedagogical guidance to students from the beginning of their studies until the end of their careers.

Keywords: Higher education, dropout, dropout prevention, dropout models.

\section{INTRODUCCIÓN}

Es sumamente importante el hecho que una sociedad tenga mayor cantidad de graduados universitarios, basándose en la teoría de Amartya Sen quien sustenta la idea de que la libertad de un pueblo es una medida para su desarrollo. Bajo esta perspectiva, se sostiene que mientras más ciudadanos altamente calificados existan, por las universidades, habrá más libertad; con mayor comprensión ética, y alta empatía de los valores sociales que sustentan una sociedad democrática (Ristoff, 2001; Adducchio et al, 2012).

Según Castillo \& Arrue, (2003) la Educación es parte de la estructura social global y constituye una herramienta valiosa para promover la movilidad en general, fortalecer la identidad y valores de la cultura nacional; acrecentar la integración social y disminuir las desigualdades de los diferentes sectores de la población. No obstante, algunos sectores de la sociedad civil consideran que las universidades no han diseñado una estrategia efectiva para lograr una vinculación exitosa con la sociedad y no se observan los beneficios que se derivan del gasto público en la educación.

Las autoras, continúan afirmando que la Educación Superior -ES- ha sido predominantemente teórica, desvinculada de los grandes, graves y complejos problemas de la sociedad actual, más preocupada por sus problemas de funcionamiento interno y no por su responsabilidad social en la construcción de una sociedad más equitativa, justa, próspera y democrática.

En ese mismo orden Martuscelli, (2012) afirma que la ES se encuentra inmersa en grandes y graves problemas tales como: insuficiente cobertura ante una demanda creciente; inequidad en el acceso en perjuicio de los jóvenes de estratos económicos bajos; limitada vinculación con los sectores productivos, desarticulación con los otros niveles educativos; apoyos insuficientes a la investigación en las universidades, débil pertinencia y calidad, crecimiento de la matrícula en instituciones particulares, deserción en los diferentes niveles y por múltiples causas. 
En Panamá las Instituciones de Estudios Superiores -IES- son atendidas tanto por el sector público o estatal; como por el sector privado. Pero el aporte del Estado para su financiamiento no crece al mismo ritmo del incremento de la matrícula y las demandas, aunque eso no impide que su cobertura involucre a toda la población que pueda asistir a la misma sin distinción de raza, credo, situación política y social (Escobar, 2005; 2006).

Las situaciones problemáticas antes mencionadas, llevan a muchos estudiantes a abandonar sus estudios sin considerar la idea de regresar y culminar su programa que una vez inició; por ejemplo, de las universidades del país, la Universidad de Panamá presenta el porcentaje de deserción más alto con 14\% seguido de la Universidad Tecnológica de Panamá con 10\% como lo muestra la tabla 1. Es importante señalar que la Universidad de Panamá es la que matricula más estudiante por año (63,091 en el año 2017).

Tabla 1: Porcentaje de estudiantes de Instituciones de Estudios Superiores (IES) públicas, que no se matricularon en el segundo semestre en la carrera inicialmente seleccionada.

\begin{tabular}{|c|c|c|c|c|c|c|}
\hline \multirow{2}{*}{ IES } & \multicolumn{6}{|c|}{ 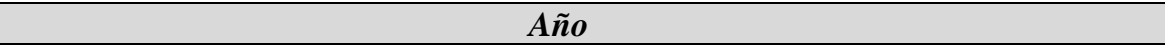 } \\
\hline & 2008 & 2009 & 2010 & 2014 & 2015 & \% promedio \\
\hline Universidad de Panamá & $17 \%$ & $15 \%$ & $14 \%$ & $12.1 \%$ & $12.6 \%$ & $14 \%$ \\
\hline $\begin{array}{l}\text { Universidad Tecnológica } \\
\text { de Panamá }\end{array}$ & $17 \%$ & $10 \%$ & $10 \%$ & $9.7 \%$ & $9.6 \%$ & $10 \%$ \\
\hline $\begin{array}{l}\text { Universidad Autónoma } \\
\text { de Chiriquí }\end{array}$ & $22 \%$ & $19 \%$ & $18 \%$ & - & - & $19 \%$ \\
\hline $\begin{array}{l}\text { Universidad Marítima } \\
\text { Internacional de Panamá }\end{array}$ & -- & -- & -- & $9.9 \%$ & $5.8 \%$ & $7 \%$ \\
\hline $\begin{array}{l}\text { Universidad } \\
\text { Especializada de las } \\
\text { Américas }\end{array}$ & $11 \%$ & $10 \%$ & $16 \%$ & $7.5 \%$ & $5.5 \%$ & $9.5 \%$ \\
\hline Total por año & $67 \%$ & $54 \%$ & $58 \%$ & $39.2 \%$ & $33.5 \%$ & \\
\hline
\end{tabular}

Fuente: Elaborada por los autores. Datos seleccionados de la sección de transparencia de las páginas web de las Universidades panameñas.

\section{METODOLOGÍA.}

En el primer momento de la investigación se diseñó, validó y aplicó un instrumento autoinstructivo denominado Cuestionario para el Análisis de la Deserción Estudiantil Universitaria CADESUN para determinar las razones por las cuales los estudiantes abandonan sus primeros años de universidad (Díaz $\&$ Tejedor, 2016).

En ese sentido, los autores citados toman en consideración Modelos de análisis de deserción para la confección del instrumento, por ejemplo: un modelo psicológico que abordan el problema desde la perspectiva individual y los rasgos de personalidad del estudiante como la causa para terminar sus estudios (Lopera Oquendo, 2008). Por otro lado, un modelo sociológico, que enfatizan las influencias de factores externos al abandono individual (Díaz Peralta, 2008).

Aunado a los modelos anteriores está el presentado por Vásquez \& Rodríguez (2007), quienes argumentan un modelo económico que adopta un enfoque de costo / beneficio que considera tanto la falta 
de recursos para cubrir los gastos de asistencia universitaria como la necesidad de trabajar o buscar empleo (Beyer, 1998; Abril Valdez et al, 2008). Un modelo organizacional enfocado, según (Donoso \& Schiefelbein, 2007), en función de los servicios que ofrecen a sus estudiantes, fundados en las cualidades de la organización educativa, la integración social entre los estudiantes y más particularmente en las experiencias de los estudiantes en el aula. Y un modelo de adaptación e integración donde se señala que la Universidad tiene un rol central en lo que respecta a la adaptación de los estudiantes a su nueva vida estudiantil y de no ser así, el estudiante deserta (Saldaña Villa, 2009), como se muestra en la Figura 1.

Figura 1: Variables conceptuales identificadas en la revisión de la literatura y utilizadas en el estudio como posibles factores determinantes de la deserción.

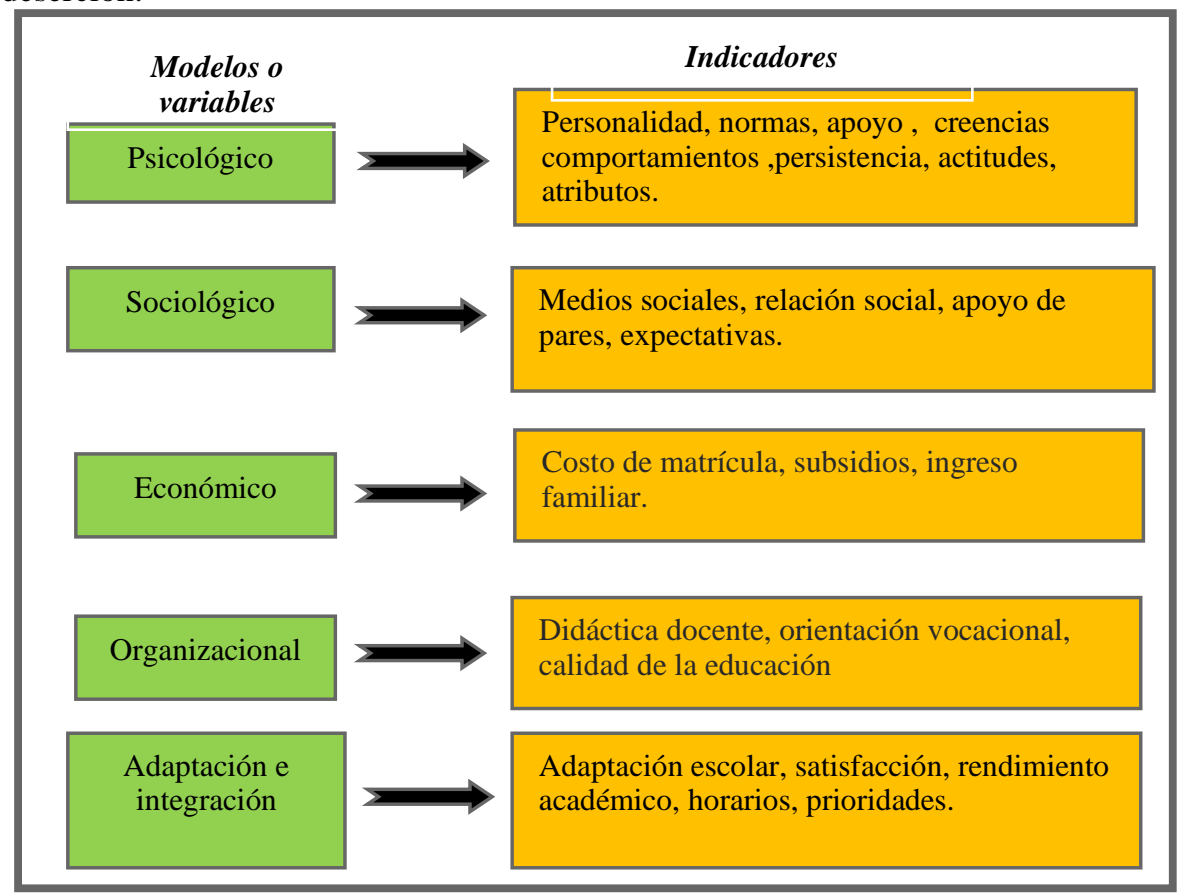

Fuente: Elaborada por Díaz \& Tejedor, (2016).

La investigación presenta un diseño no experimental porque no hay condiciones ni estímulos a los cuales se hayan expuesto los sujetos. Para el estudio de la deserción estudiantil en las IES, la literatura que se consultó hace referencia a una serie de modelos conductuales que tratan de responder al fenómeno educativo, por lo que ha desarrollado un estudio de tipo descriptivo y analítico (Díaz \& Tejedor, 2017).

El instrumento CADESUN se basó en los planteamientos de Braxton et al, (2000) \& Himmel (2002), haciéndose énfasis solamente en la dimensión estudiantil, o sea, un enfoque de variable individual, ya que los autores también plantean la posibilidad de incluir la dimensión de los gestores de la administración, de los tutores o entorno familiar. Se evaluaron los estudiantes desertores matriculados (primer ingreso) en los años 2014 y 2015 en una IES pública. La población consta de 214 desertores para los años correspondientes y la muestra de 138. Los estudiantes fueron seleccionados con un tipo de 
muestra probabilística, aleatoria simple y por reposición. Para el análisis de los resultados se utiliza el estadígrafo ANOVA.

\section{ANÁLISIS DE LOS RESULTADOS.}

En el presente estudio se muestran los resultados derivados de la implementación del CADESUN, de Díaz \& Tejedor, (2016) analizado factor por factor para contextualizar el instrumento al análisis global de los cinco factores asociados a la deserción y tratar de identificar qué tipo de modelo fue el seguido por la población estudiantil desertora estudiada. Cabe señalar que, para conocer si un factor incide más que

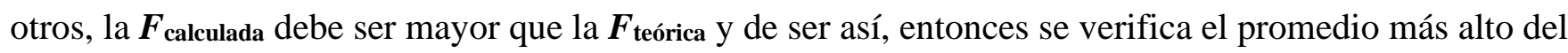
factor o subfactor tomado en consideración.

Para valorar el modelo Psicológico, según Díaz \& Tejedor, (2016) específicamente los rasgos de la personalidad y su incidencia en la deserción, se recurrió (ANOVA) análisis de varianza de un solo factor para sub-factores simultáneos ( $\mathbf{F}$ de Fisher) para un coeficiente de confiabilidad de 0.05 ( $<<0.05)$ donde se determinó que $\boldsymbol{F}_{\text {calculada }}$ (15.18) fue mayor que la $\boldsymbol{F}_{\text {teórica }}(2.02)$ para un alfa $(\alpha)$ de 0.05 ; lo cual es indicativo de que los ocho sub-factores que comprenden al factor psicológico tienen un episodio significativo diferenciado sobre la deserción y siguen el comportamiento de incidencia:

Normas $>$ Apoyo $>$ Comportamientos $>$ Persistencia $>$ Creencias $>$ Actitudes $>$ Personalidad $>$ Atributos.

De la distribución anterior se observa que lo relacionado con el subfactor Normas, con un promedio de (3.17) y con una varianza de (2.14) es el sub factor, dentro del factor psicológico, que más incide en el fenómeno de la deserción. O sea que los estudiantes de la IES no estaban de acuerdo con las normas y leyes universitaria, por lo que decidieron desertar.

Para el factor Sociológico que contemplaron cuatro sub-factores (medios sociales, relación social, apoyo de pares, expectativas) y el factor Económico y sus tres sub-factores (costos de matrícula, los subsidios y los ingresos familiares), aplicado el estadígrafo de análisis no se pudieron detectar diferencias significativas entre los sub-factores estudiados, es decir, que incidien en igual proporción en la deserción. En estos casos la $\boldsymbol{F}_{\text {calculada }}$ fue menor que la $\boldsymbol{F}_{\text {teórica. }}$

El modelo organizacional enfoca la deserción desde las características de la institución universitaria, a la integración social entre estudiantes, dentro del salón de clases; a las consultas de los estudiantes y a la orientación vocacional que recibió. Realizado el análisis estadístico se pudo verificar que para el factor organizacional la $\boldsymbol{F}_{\text {calculada }}(3.45)$ fue mayor que la $\boldsymbol{F}_{\text {teórica }}(2.39)$, lo que indica que los cinco sub-factores que comprenden este factor, tienen una incidencia significativa diferenciada sobre la deserción y siguen el comportamiento mostrado: 
Actividades de apoyo académico > Consultorías > Orientación vocacional > Didáctica del docente > Calidad de la educación.

De la distribución anterior se observa que la relación con las Actividades de apoyo académico es el sub factor, dentro del modelo organizacional que más incidió en la deserción de los estudiantes. Lo anterior se refuerza por las declaraciones manifestadas por algunos estudiantes: "son muy precarios, los servicios de apoyo académico- administrativos (bibliotecas, librería, servicios médicos, orientación profesional y vocacional.), que brinda la institución a sus estudiantes”.

Al considerar los valores promedios a las respuestas del factor Adaptación e Integración y sus subfactores, y aplicado el estadígrafo de análisis se determinó que para el factor Adaptación e Integración sus (9) sub-factores inciden diferencialmente en la deserción y lo hacen de la siguiente manera:

Adaptación > Acceso > Estado emocional = Horario > Satisfacción > Aspiraciones > Prioridades > Interacción > Esfuerzo.

Es oportuno destacar, que debido a la inadecuada Adaptación de los estudiantes a su nuevo rol dentro el ambiente universitario, fue un factor decisivo para que este abandonara sus estudios al finalizar el primer semestre de la carrera. Hallazgos similares fueron reportados por Yengle Ruiz, (2009) quien considera que las dificultades de adaptación a la vida estudiantil universitaria, durante los primeros ciclos, constituye un factor esencial asociada a la deserción.

Díaz \& Tejedor (2016) sostienen que el CADESUN fue diseñado con el propósito de servir como instrumento de análisis para determinar la existencia o no de un modelo patrón seguido por los estudiantes desertores de una carrera universitaria.

Por tal razón, al analizar los resultados obtenidos de la población desertora y partiendo de la hipótesis nula donde plantó que el CADESUN no era capaz de identificar un tipo particular de modelo de deserción, se realizó un análisis de varianza de un solo factor para factores simultáneos ( $\boldsymbol{F}$ de Fisher) y se determinó que la $\boldsymbol{F}_{\text {calculada }}(21.60)$ fue mayor que la $\boldsymbol{F}_{\text {teórica }}(2.38)$ para un alfa $(\alpha)$ de 0.05 ; lo que comprueba que la deserción de los estudiantes de la Extensión universitaria estudiada siguió, en efecto, un modelo típico de comportamiento como lo muestra la Tabla 1.

$\mathrm{Al}$ respecto, se tomaron en consideración los valores promedios globales a las respuestas obtenidas y ordenados de mayor a menor, el orden de incidencia de los cinco factores sigue la siguiente distribución:

Económico > Organizacional > Adaptación e Integración > Psicológico > Sociológico.

Por lo que se interpreta, que la deserción manifiesta por los estudiantes seleccionados tiende a comportarse según el Modelo Económico.

Díaz \& Tejedor (2016) argumentan que los resultados de investigaciones similares realizadas por Sánchez-Amaya et al, (2009), afirman que cuando las economías familiares son frágiles es difícil 
pretender un apoyo económico sostenible a lo largo de toda la carrera para el mantenimiento del estudiante, pues las necesidades de la familia priorizan el trabajo por el estudio.

Tabla 1: Prueba $F$ de Fisher (ANOVA) para los Modelos de análisis de la deserción estudiantil.

\begin{tabular}{l|c|c}
\hline \multicolumn{1}{c|}{ Modelos } & Promedio & Varianza \\
\hline Psicológico. & 1.98 & 1.65 \\
Sociológico. & 1.80 & 1.28 \\
Económico. & 2.59 & 2.53 \\
Organizacional. & 2.21 & 1.92 \\
Integración y adaptación. & 2.00 & 1.76 \\
\hline
\end{tabular}

\begin{tabular}{l|c|c|c|c}
\hline \multicolumn{1}{c|}{ Origen de las variaciones } & $\begin{array}{c}\text { Suma de } \\
\text { cuadrados }\end{array}$ & $\begin{array}{c}\text { Grados de } \\
\text { libertad }\end{array}$ & $\boldsymbol{f}_{\text {calculada }}$ & $\boldsymbol{f}_{\text {teórica }}$ \\
\hline Entre los sub-factores & 158.46 & 4 & & \\
Dentro de los sub-factores & 5090.30 & 2793 & 21.73 & 2.38 \\
Total & 5248.76 & 2797 & & \\
\hline
\end{tabular}

Fuente: Elaborada por los autores y tomando como referencia los datos del estadígrafo ANOVA.

En el segundo momento de la investigación presentada se trata de diseñar, validar y poner en práctica un programa de prevención de deserción estudiantil que permita a las universidades medir y monitorear, factores determinantes de deserción, conocer su evolución en el tiempo y tomar medidas de prevención, de sensibilización y acciones de apoyo a los estudiantes Backes \& Zavala, (2020).

Esta propuesta parte de una primera fase de identificación y análisis de diversos sistemas, modelos o programas de prevención de deserción como de fomento de la permanencia en las IES, una segunda fase de diseño y validación de un programa de prevención basados en Modelos Latinoamericanos apoyado en datos específicos para tal fin, una tercera fase de sensibilización sobre el implemento del programa de prevención de deserción estudiantil a la comunidad educativa. Una cuarta fase que se refiere a la puesta en práctica del programa de prevención a los estudiantes que se consideren en peligro de desertar (grupos experimentales). Una quinta fase que tiene que ver con la evaluación del programa por expertos acompañado con el seguimiento y orientación constante a los estudiantes que lo requieran.

\section{CONCLUSIONES}

Existe un significativo porcentaje de deserción en las universidades públicas del país, y específicamente en la Extensión Universitaria seleccionada en la provincia Veraguas; donde los y las estudiantes que desertan, se caracterizan por ser jóvenes que abandonan las instituciones de estudios especialmente durante los primeros semestres.

La mayor razón de abandono estudiantil de la IES seleccionada es la Económica, que incluye el costo de la matricula que afecta la estadía, los subsidios que brindan algunas instituciones, como también 
los ingresos familiares para poder enfrentar los gastos que demanda la asistencia a una Institución de Estudios Superiores (IES).

Los modelos expuestos, para el análisis de los resultados que incluyen diversas dimensiones e indicadores; han logrado determinar la medida en que dichos factores inciden de una forma u otra en la deserción estudiantil en la educación superior.

\section{REFERENCIAS}

Abril Valdez, E.; Román Pérez, R.; Cubillas Rodríguez, M.J. \& Moreno Celaya, I. (2008). ¿Deserción o autoexclusión? Un análisis de las causas de abandono escolar en estudiantes de educación media superior en Sonora, México. Revista Electrónica de Investigación Educativa. v. 10, n. 1, p. 1-16.

Adducchio, S.P.; Escobar, P.M., Larrosa, J.M. \& Salvio, M.J (2012). Proyecto Mutual de Crédito Universitario. Buenos Aires: Universidad Nacional de La Matanza, 82 p.

Backes Dos Santos, K.N. \& Zavala Saucedo, M.V. (2020). Participation of women in management positions in an institution of higher education of public management-Ciudad Del Este -Paraguay. South Florida Journal of Develoupment. V.2, n.1, p. 145-154.

Beyer, H. (1998). ¿Desempleo juvenil o un problema de deserción escolar? Estudios Públicos. n. 71, p. 89 - 119.

Braxton, J.M.; Milem, J.F. \& Sullivan, A. (2000). The influence of active learning on the college student departure process. The Journal of Education. v. 71, n. 5, p. 569 - 590.

Castillo, N. \& Arrue, Á. (2003) Informe Nacional de Educación Superior de Panamá. Consejo de Rectores de Panamá: Universidad Tecnológica de Panamá. 135 p.

Díaz, P. y Tejedor, A. (2016) Design and Validation of a Questionnaire to Analyze University DropoutCADES. World Journal of Educational Research. v. 3, n. 2, p. 267-280.

Díaz, P. y Tejedor, A. (2017) El CADESUN, Un nuevo instrumento para analizar la deserción estudiantil universitaria. Revista Reencuentro. v. 73, p. 199-215.

Donoso, S. \& Schiefelbein, E. (2007). Análisis de los modelos explicativos de retención de estudiantes en la universidad: una visión desde la desigualdad social. Estudios Pedagógicos. v. 33, n.1, p. 7 - 27.

Lopera Oquendo, C. (2008) Determinantes de la deserción universitaria en la facultad de economía universidad del Rosario. Borradores de Investigación. n. 95, p.25.

Escobar, V. de. (2005). Estudio sobre la deserción y repitencia en la educación superior en Panamá. Panamá: Instituto Internacional para la Educación Superior En América Latina y El Caribe. 31 p.

Escobar, V. de. (2006) Informe de la educación superior en Panamá. Estudio para CINDA UNIVERSIA Subdirección de Evaluación Universitaria de la Universidad de Panamá. 44 p. 
Himmel, E. (2002) Modelos de análisis de la deserción estudiantil en la educación. Calidad en la Educación. p. 91 - 108.

Martuscelli, J. (2012) Problemas más urgentes en la educación superior. Universidad Autónoma de México 14 Diapositivas, color azul. Disponible: <http://es.scribd.com/doc/99136557/6-MartuscelliProblemas-Educacion Superior>. Acceso: 22/ene/12.

Ristoff, D.I. (2001). O exame nacional de curso e a avaliação institucional. Avaliação: Revista da Rede de Avaliação da Educação Superior. v. 6, n. 3 (21), p. 21 - 29.

Saldaña Villa, M.C. (2009) Perfil del alumno desertor en la Universidad Católica de la Santísima Concepción: un estudio de caso.129 h. Disertación (Maestría) Departamento de Sociología y Antropología. Facultad de Ciencias Sociales Universidad de Concepción, Chile.

Sánchez Amaya, G.; Navarro Salcedo, W. \& García Valencia, A.D. (2009). Factores de deserción estudiantil en la Universidad Surcolombiana, Sede Neiva 2002-2005. Revista Paideia Surcolombiana. p. $97-103$.

Vásquez Martínez, C.R. \& Rodríguez Pérez, M.C. (2007). La deserción estudiantil en educación superior a distancia: perspectiva teórica y factores de incidencia. Revista Latinoamericana de Estudios Educativos. v. 37, n. 3-4, p. 107 - 122.

Yengle Ruiz, C. (2009). Adaptación a la vida universitaria de estudiantes que desertaron asociada a la relación con compañeros de estudio. Scientia. v. 1, n. 1, p. 40 - 50. 